\section{Variable manufacturing characteristics of the P-15 phosphor: A warning}

\section{WILLIAM R. UTTAL \\ University of Michigan, Ann Arbor, Michigan 41804}

In the last few years, our laboratory has been pursuing a research program with computer-controlled oscilloscopes as the display device (see, for example, Uttal, 1971). This instrumentation system feeds all its computational output into a microscopic layer of phosphorescent material on the face of the cathode ray tube (CRT) in the oscilloscope. We had specifically chosen the P-15 phosphor for our studies because of its very brief persistence. According to the manufacturer, the light output from this phosphor decays to less than $1 / 10$ of $1 \%$ of the original brightness in about 50 microsec. Ultrabrief persistences of this sort were chosen so that any interaction between sequential signals would be a function of the much longer persistence of the visual system within the head of the $S$ rather than the persistence of the phosphor's output light from the oscilloscope.

Up until the last few months, we had been using Tektronix Type 561A - oscilloscopes (one in the rack-mounting configuration and one in the ordinary cabinet configuration) as the display unit in our experiments. Over a period of 6 years, we have run a large number of experiments which have led to a reasonably good standardization of the results which could be expected to be obtained under certain standard conditions. During the last year or so, the instruments which had served us so faithfully have begun to falter. Controls have become noisy, CRTs have become worn, and most seriously, stability in brightness and position has become increasingly difficult to maintain. We have also become increasingly aware of the heat problem generated by these vacuum tube oscilloscopes as additional equipment has been added to our laboratory.

We therefore decided to update our display units by ordering and installing transistorized Tektronix Type 602 oscilloscopes, which are more specifically designed for point-plotting procedures. These instruments were ordered with the same P-15 phosphor we had so successfully been using for so long.

Upon arrival, it became immediately apparent that the results with the two oscilloscopes were not the same. We found, for example, that identical conditions led to differences as great as $12 \%$ in correct recognition of a dotted pattern embedded in bursts of dotted random noise when the same experiments were carried out on the old (higher scores) and new (lower scores) oscilloscopes. Repeated trials of the same condition on the old oscilloscope, or on the new one, however, resulted in reliability within a couple of percentage points.

We have explored a number of technical possibilities which might have explained the difference between the two instruments. The dot size on the Type 602 oscilloscope is smaller than that of the Type 561A. Defocusing the dot on the 602 produced a dot comparable to that of the $561 \mathrm{~A}$ without, however, mitigating the difference with which we were confronted. Similarly, variation of the dot brightness over wide ranges could not account for the difference, nor could various manipulations of the mounting geometry of the oscilloscope. As we had previously observed in a number of different contexts, there are only minimal effects of either visual ángle or spot brightness in this task, which appears to be mainly mediated by signal-to-noise considerations between the target and the masking noise dots.

Two possible explanations thus remain: There may be some difference in the electronics of the new oscilloscope, or (perhaps more likely) there may be some difference in the phosphor which accounts for the difference in masking results. In either case, the display unit itself seems to be able to inject into the experiments a variability of a sort of which we were not aware and which is for all practical purposes uncontrollable. We believe the difference actually resides in changes in the property of the phosphor itself.

To use the new oscilloscopes in our experiment, we now have had to shift the standard range of noise dot density over which our Ss display continuous variability. This is a satisfactory procedure for our purposes, since in all of our experiments there is always an internal comparison made between some standard condition and some other range of variation of the independent variable. However, attempts to replicate the exact numerical scores reported in our earlier studies with the older oscilloscopes would necessarily be confounded by this variability in what we believe to be the manufactured characteristics of the phosphor.

Though we have not been able to pin down the exact cause of this difference, it is definitely in the oscilloscope and probably in the phosphor, and future users of this equipment should be aware of the variability inherent in older and newer versions of this sort of display. Since the results with two old 561A oscilloscopes are compatible with each other, it seems unlikely that this difficulty is due to wide variability among individual oscilloscopes, but rather that there has been some change in the manufactured characteristics of the phosphor over the years. Other researchers may wish to consider this variability, whether due to phosphor or some other controlling characteristic, as the use of computer-controlled oscilloscopes becomes more widespread and attempts to standardize or replicate experiments become more common.

\section{REFERENCE}

Uttal, W. R. The effect of interval and numbex on masking with dot bursts. Perception \& Psychophysics, 1971, 9, 469-473.

(Received for publication October 20, 1972; accepted October 27, 1972.) 